\title{
ON SIMULTANEOUS APPROXIMATION AND INTERPOLATION WHICH PRESERVES THE NORM
}

\author{
BY FRANK DEUTSCH ${ }^{1}$ AND PETER D. MORRIS ${ }^{1}$
}

Communicated by Avner Friedman, February 14, 1969

In [6] H. Yamabe established the following "simultaneous approximation and interpolation" theorem, which generalized a result of Walsh [4, p. 310] (cf. also [1], [3] for further generalizations), and is related to a theorem of Helly in the theory of moments (cf. e.g. [2, pp. 86-87]).

TheOREM (YAMABE). Let $M$ be a dense convex subset of the real normed linear space $X$, and let $x_{1}^{*}, \cdots, x_{n}^{*} \in X^{*}$. Then for each $x \in X$ and each $\epsilon>0$, there exists a $y \in M$ such that $\|x-y\|<\epsilon$ and $x_{i}^{*}(y)$ $=x_{i}^{*}(x)(i=1, \cdots, n)$.

Wolibner [5], in essence, proved that Yamabe's theorem could be sharpened in the particular case when $X=C([a, b]), M=\mathcal{P}=$ "the polynomials," and the $x_{i}^{*}$ are "point evaluations." Indeed, from the results of [5] there can readily be deduced the following

Theorem (Wolibner). Let $a \leqq t_{1}<\cdots<t_{n} \leqq b$ and let $P$ be the set of polynomials. Then for each $x \in C([a, b])$ and each $\epsilon>0$, there exists a $p \in \mathcal{P}$ such that $\|x-p\|<\epsilon, p\left(t_{i}\right)=x\left(t_{i}\right)(i=1, \cdots, n)$, and $\|p\|=\|x\|$.

Motivated by Wolibner's theorem, we consider the following more general problem. Let $M$ be a dense subspace of the real normed linear space $X$, and let $\left\{x_{1}^{*}, \cdots, x_{n}^{*}\right\}$ be a finite subset of the dual space $X^{*}$. The triple $\left(X, M,\left\{x_{1}^{*}, \cdots, x_{n}^{*}\right\}\right)$ will be said to have property $S A I N$ (simultaneous approximation and interpolation which is norm-preserving) provided that the following condition is satisfied:

For each $x \in X$ and each $\epsilon>0$ there exists a $y \in M$ such that $\|x-y\|$ $<\epsilon, x_{i}^{*}(y)=x_{i}^{*}(x)(i=1, \cdots, n)$, and $\|y\|=\|x\|$.

In this note we shall outline some of the main results we have obtained regarding property SAIN. Detailed proofs and related matter will appear elsewhere.

Theorem 1. Let $M$ be a dense subspace of the Hilbert space $X$ and let $x_{1}^{*}, \cdots, x_{n}^{*} \in X^{*}$. Then $\left(X, M,\left\{x_{1}^{*}, \cdots, x_{n}^{*}\right\}\right)$ has property $S A I N$ if and only if each $x_{i}^{*}$ attains its norm on the unit ball in $M$.

${ }^{1}$ Supported by grants from the National Science Foundation. 
The necessity in Theorem 1 is valid in any reflexive Banach space $X$. Whether the sufficiency is also valid in any reflexive Banach space is an open question. Also, in the case when $n=1$, Theorem 1 is valid in any strictly convex reflexive Banach space.

Let $T$ denote a compact Hausdorff space and $C(T)$ the real continuous functions on $T$ with the sup norm. If $t \in T, \delta_{t}$ will denote the functional "point evaluation" at $t$, i.e. $\delta_{t}(x)=x(t)$ for all $x \in C(T)$.

Theorem 2. Let $A$ be a dense subalgebra of $C(T)$ and $t_{1}, \cdots, t_{n} \in T$. Then $\left(C(T), A,\left\{\delta_{t_{1}}, \cdots, \delta_{t_{n}}\right\}\right)$ has property $S A I N$.

Theorem 2 contains that of Wolibner and represents a strengthening of the Stone-Weierstrass theorem. Theorem 2 is proved by a rather tedious induction on $n$ using Yamabe's theorem and the following lemma which essentially allows us to approximate the unit function in a useful manner.

Lemma. Let $A$ and $t_{i}$ be as in Theorem 2. Then for each $\epsilon>0$, there exists an element $e \in A$ such that $\|e-1\|<\epsilon, e\left(t_{i}\right)=1(i=1, \cdots, n)$, and $e \leqq 1$.

Theorem 2 is also valid if "dense subalgebra" is replaced by "dense linear sublattice containing constants." However, the following examples show that these results cannot be extended very far.

EXAMPLE 1. Let

$$
M=\left\{x \in C([0,1]): x^{\prime}\left(\frac{1}{2}\right) \text { exists, } x^{\prime}\left(\frac{1}{2}\right)=x(0)-x(1)\right\} .
$$

Then $M$ is a dense subspace of $C([0,1])$, which contains constants, but such that $\left(C([0,1]), M, \delta_{1 / 2}\right)$ does not have property SAIN (since if $x \in C([0,1])$ is the function which is 1 if $0 \leqq t \leqq \frac{1}{2}$ and $x(t)=-2 t+2$ if $\frac{1}{2}<t \leqq 1$, and $y$ is any element of $M$ which satisfies $y\left(\frac{1}{2}\right)=x\left(\frac{1}{2}\right)=1$ and $\|y\|=\|x\|=1$, then $y^{\prime}\left(\frac{1}{2}\right)=0$ so $y(0)=y(1)$ and hence $\left.\|x-y\| \geqq \frac{1}{2}\right)$.

EXAMPLE 2. Let $A=\operatorname{span}\left\{x_{1}, \quad x_{2}, \cdots\right\}$ where $x_{i}(t)=t^{i}$ $(i=1,2, \cdots)$ and define $x^{*}$ by $x^{*}(x)=\int_{1}^{2} x(t) d t$ for all $x \in C([1,2])$. Then $A$ is a dense subalgebra of $C([1,2])$ but $\left(C([1,2]), A, x^{*}\right)$ does not have property SAIN (since if $e$ is the unit function, then any $y \in A$ which satisfies $x^{*}(y)=x^{*}(e)=1$ must necessarily satisfy $\|y\|>1=\|e\|)$.

EXAMPLE 3. Let

$$
L=\left\{x \in C([0,1]): x^{\prime}(0) \text { exists, } x^{\prime}(0)=x(0)\right\} .
$$

Then $L$ is a dense linear sublattice in $C([0,1])$ but $\left(C([0,1]), L, \delta_{0}\right)$ does not have property SAIN (since if $e$ is the unit function and $y$ is any element of $L$ satisfying $y(0)=e(0)=1$, then $y^{\prime}(0)=y(0)=1$ and 
so $y(t)>1$ for some $t>0$ and hence $\|y\|>1=\|e\|)$.

In the case when $X=L_{p}=L_{p}(T, \Sigma, \mu)(1<p<\infty)$ and $M$ is the subspace of $L_{p}$ consisting of those functions which vanish off sets of finite measure, we can prove the following theorem. (Recall that the representer of a functional $x^{*} \in L_{p}^{*}$ is the function $y \in L_{q}, q=p /(p-1)$, such that $x^{*}(x)=\int_{T} x y d \mu$ for all $x \in L_{p}$.)

Theorem 3. Let $1<p<\infty$, let $M \subset L_{p}$ be as above, and let $x_{1}^{*}, \ldots$, $x_{n}^{*} \in L_{p}^{*}$. Then the following statements are equivalent.

(1) $\left(L_{p}, M,\left\{x_{1}^{*}, \cdots, x_{n}^{*}\right\}\right)$ has property $S A I N$.

(2) Each $x_{i}^{*}$ attains its norm on the unit ball in $M$.

(3) The representer of each $x_{i}^{*}$ vanishes off a set of finite measure.

\section{REFERENCES}

1. F. Deutsch, Simultaneous interpolation and approximation in linear topological spaces, SIAM J. Appl. Math. 14 (1966), 1180-1190.

2. N. Dunford and H. T. Schwartz, Linear operators. Part I: General theory, Interscience, New York, 1958.

3. I. Singer, Remarque sur un thêorème d'approximation de H. Yamabe, Atti Accad. Naz. Lincei Rend. Cl. Sci. Fis. Mat. Natur. 26 (1959), 33-34.

4. J. L. Walsh, Interpolation and approximation by rational functions in the complex domain, Amer. Math. Soc. Colloq. Publ., vol. 20, Amer. Math. Soc., Providence, R. I., 1935.

5. W. Wolibner, Sur un polynôme d'interpolation, Colloq. Math. 2 (1951), 136137. 15-17.

6. H. Yamabe, On an extension of the Helly's Theorem, Osaka J. Math. 2 (1950),

The Pennsylvania State University, University Park, Pennsylvania 16802 